\title{
Human Safety Algorithms for a Parallel Cable-Driven Haptic Interface
}

\author{
Martin J.-D. Otis, Sylvain Comtois, Denis Laurendeau and Clément Gosselin
}

\begin{abstract}
A parallel cable-driven haptic interface is designed to allow interaction with any type of virtual object. This paper presents and analyzes computational methods for addressing the issues regarding human safety and control reliability using such an interface, thereby ensuring safe operations inside the virtual world. Four strategies are explored: sensor reliability, mechanical interference management, workspace management and human-robot interaction. This paper focuses mainly on the sensors' reliability and workspace management algorithms for a parallel cable-driven haptic interface that imposes special requirements on the control architecture design. One challenging task is to develop efficient computational algorithms for hard real-time processes included in haptic display applications which improve safety without compromising performance.
\end{abstract}

\section{Introduction}

A Cable-Driven Locomotion Interface (CDLI) has been proposed as a haptic interface to be used in applications such as training in virtual environments [8]. This CDLI is composed of two cable-driven haptic platforms (or, in the following, the cable-driven haptic interface), one for each foot, controlled with eight cables acting as mechanical transmission. A user can walk inside a virtual environment and interact with any objects in it, including the environment itself. Due to the geometry of cable-driven haptic platforms described in [13] and the trajectories of the feet, cable interference can occur, and the platform can leave its designated workspace. Furthermore, when in use, cables can snap, wind up incorrectly on their reels, or damage their tension sensors, all potentially leading to failure.

M. J.-D. Otis, S. Comtois and D. Laurendeau

Computer Vision and Systems Laboratory, University Laval, Québec

C. Gosselin

Robotics Laboratory, University Laval, Québec e-mail: gosselin@gmc.ulaval.ca 
In the context of a human and a mechanism interacting within the same workspace, the safety of the user is an issue of utmost importance to avoid accidents and injuries. Because it isn't always safe to power-down the mechanism in hazardous situations, the main control algorithm is coupled with a safety manager using an error handler that was designed based on a risk analysis study. For example, the HapticWalker locomotion interface with permanent foot fixture has a safety manager [17]. However, its safety manager could be improved for a cable-driven haptic interface, since cables can interfere with one another or even sever. An analysis of cable interferences is presented in [19] and fault tolerance for a kinematically redundant manipulator is presented in [15]. Yet avoiding cable interference with path-planning such as in [7] is not always feasible since the user movement could be completely random. These two studies are used for managing cable fatigue and interference for enhancing safety.


Fig. 2 Full view of the scaled version of the Fig. 1 Human safety and Security Management CDLI showing the platforms, the cables and the Plan

Virtual Reality screen displaying the scene

Each component of the software must have self-testing capabilities labelled BuiltIn Self Test (BIST) describing a general system test planning for the purpose of quality assurance and safety management. A Hardware-in-the-loop (HIL) simulator could be implemented as a mean of running some parts of the BIST and partially control the platform. Fig. 1 explains the test plan implemented inside the controller for the Cable-Driven Haptic Interface presented in this paper.

This paper addresses modern human safety concerns applied to a parallel cabledriven haptic interface. The approach is itemized as four simple algorithms to enhance human safety. The safety manager defines new modes of operations for a cable-driven haptic mechanism which are investigated thoroughly: 1) critical runtime error (which should never occur); 2) soft run-time error; 3) sensor fault; 4) released cable from an active actuation state; and 5) normal mode. Furthermore, three articular control levels are implemented with an hybrid position/tension control in open and closed-loop. 
The main issue presented in this paper is the management of the platform when it leaves its workspace (generated by sensor faults, cable interference and when the platform is forced to go beyond the boundary of its usual workspace) since a defective reel that must be released from an active actuation will modify the workspace size. Indeed, the haptic application is not suitable for this critical situation where safety is more important than rendering virtual objects.

The first section of this paper presents the overall control algorithm of the parallel cable-driven haptic interface with the definition of an Optimal Tension Distriution algorithm designed for redundant actuation. The second section presents the strategies for avoiding user injury. They provide some simple methods of reducing the hazards of human-robot interaction. Finally, since the system is to provide a locomotion interface as a haptic device, the algorithms must be transparent to the user. Thus, the last section presents some results on the scaled-down $1: 3$ prototype of the Cable-Driven Locomotion Interface which is shown in Fig. 2. For demonstration, a Kondo KHR-1HV humanoid robot is installed on the platforms.

\section{Control Algorithm}

In a gait training application, the mechanism can be used to render various ground properties which can potentially reach fatal levels. Some safety algorithms and hardware systems are designed inside the mechanism gait trainer named HapticWalker [17]. The controller presented in this section comes from the idea of Lafourcarde [6] and is an extension of the HapticWalker for a parallel cable-driven interface. Other safety algorithms are presented in [18] which allow human operators and robots to work in close proximity for assisted collaboration. They use vision systems to measure body posture and position and to avoid collisions contrary to the project in [3] where collisions are allowed such that the user can push the robot intuitively (even though the robot is still under position control). In mobile robotics, the safety manager detect hazardous characteristics, evaluate them and act to avoid them [11]. Such a manager could be applied for a gait trainer where cable interference could be a hazardous characteristic.

The software architecture is divided in seven stages as defined in Fig. 3: 1) the testing simulator (BIST) with the safety manager; 2) the haptic rendering [8]; 3) the 4-DOF washout algorithm such as those suggested in $[5,12,20]$; 4) the analysis of stability [4]; 5) the cable interference management algorithm with the WrenchClosure Workspace [10]; 6) the Optimal Tension Distribution algorithm; and 7) the cable tension controllers including the motorized reels and the power amplifiers. The Wrench-Closure Workspace (WCW), defined in [2], gives the set of poses of the moving platform for which any external wrench can be balanced by positive tensions in the cables. Vector $\mathbf{F}$ represents a flag indicating when the evaluated Cartesian pose $\mathbf{P}_{p f}$ is inside this workspace which will be used in the algorithm 4.

Fig. 1 presents the architecture of the safety design implemented in the safety manager presented in Fig. 3. The design priority goes from the center to the outside 


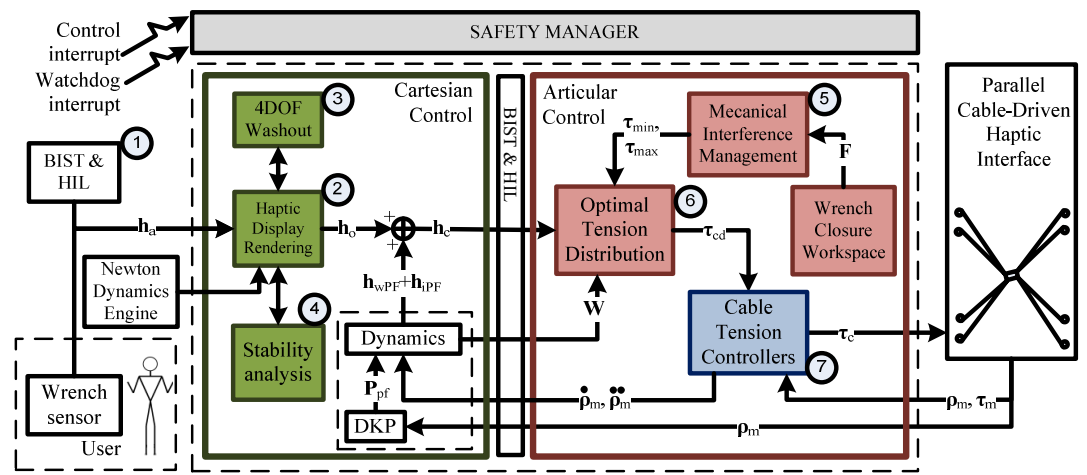

Fig. 3 Simplified control system of the parallel cable-driven haptic interface

of Fig. 1. A fault in the smaller circles could generate a fault in the bigger ones (e.g., a damaged reel changes the platform workspace dimensions). However, a fault in the outer circles does not affect the inner ones. Also, the algorithms are not competitive: whether or not cable interference occurs and the platform leaves its workspace, the software continues its safe operation.

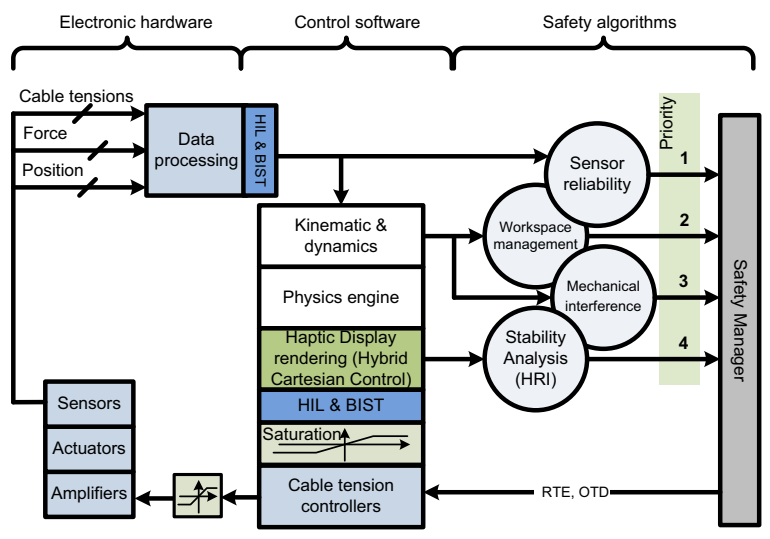

Fig. 4 Implementation of the safety algorithms

An implementation level is shown in Fig. 4 for each safety algorithms with their associated execution priority. Algorithm 1 is the main loop inside the controller where $\mathbf{T}_{c d}$ is a circular buffer of length $l$ containing the previous cable tension values $\tau_{c d}, m$ is the index of the actual values and $\mathbf{E}$ represents any errors generated inside the algorithms and inside any functions. This main algorithm presents the calling order for each stage inside the control system which corresponds to a particular 
case of the Fig. 4 where each safety algorithms with execution priority levels are described.

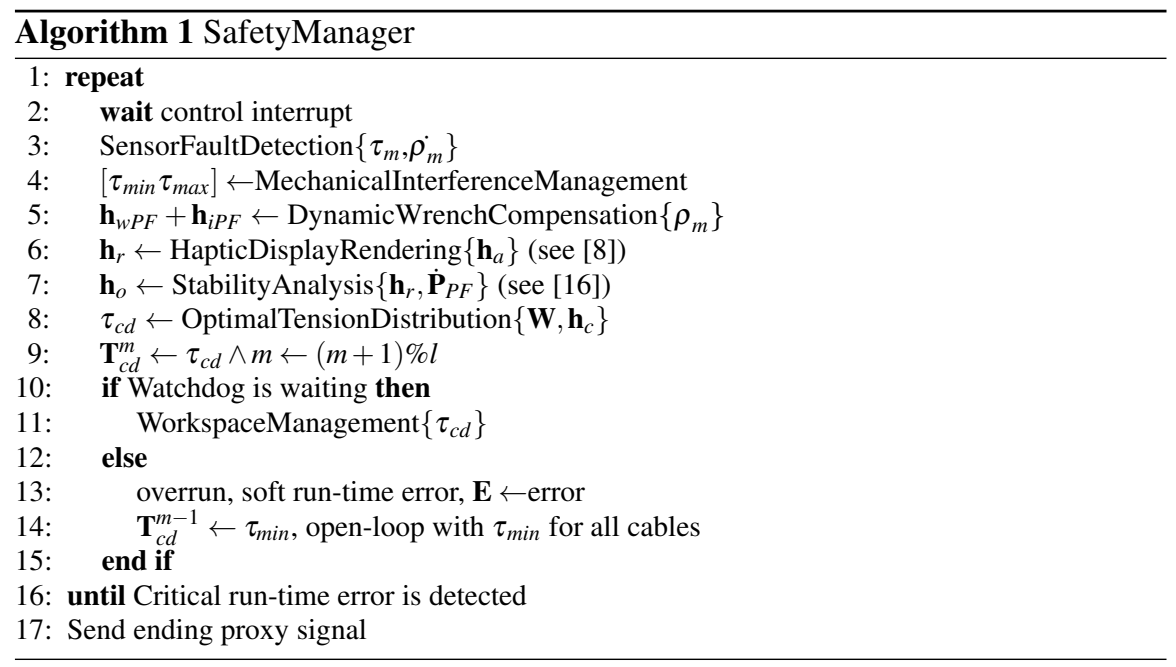

\subsection{Optimal Tension Distribution (OTD)}

Optimal Tension Distribution (OTD) generates a set of equilibrium tension values called the setpoints that the cable tension controllers then attempt to follow [21]. Cable sagging is reduced by maintaining the tension in the cables above a minimum threshold value $\tau_{\min }$. Indeed, values of $\tau_{\min }$ are not constants and can be computed with the cable length and the attachement points on the platform. The actuation system (reels, motors and cables) is also limited by a maximum tension $\tau_{\max }$. Hence, the following force distribution method in (1) is proposed to avoid cable sagging as well as excessive mechanical deformation of the reel:

$$
\begin{aligned}
& \left\{\begin{array}{l|l}
\text { minimize } & \multicolumn{1}{c}{\frac{1}{2} \tau_{c d}^{T} \mathbf{G} \tau_{c d}} \\
\text { under } & \mathbf{W} \tau_{c d}=\mathbf{h}_{c}, \text { with } \\
& \tau_{\text {max }} \geq \tau_{c d}[i] \geq \tau_{\min }
\end{array}\right. \\
& \mathbf{G}=\quad \operatorname{diag}\left(g_{i}\right) \\
& \mathbf{h}_{c}=\mathbf{h}_{w P F}+\mathbf{h}_{i P F}+\mathbf{h}_{o} \\
& \tau_{c d}=\left[\begin{array}{llll}
\tau_{1} & \tau_{2} & \ldots & \tau_{n}
\end{array}\right]^{T} \\
& i=0,2, \ldots, n-1 \text { cables, }
\end{aligned}
$$

where $\mathbf{h}_{c}$ represents the forces and torques that are applied on a single platform (i.e. the total wrench applied by the cables at the centre of mass of that platform), 
$\tau_{c d}[i]$ is the tension vector of the $\mathrm{i}$ th (of $\mathrm{n}$ ) cable, $\mathbf{W}$ is the pose-dependent wrench matrix computed by the platform Jacobian matrix that links Cartesian to articular velocities, $\mathbf{G}$ is a weighting matrix with its diagonal elements such that $g_{i}=1$ for all $i$. The mathematical derivation of (1) is presented in [1].

The effect of a broken cable, a defective sensor or a released cable is equivalent to removing a column in the matrix $\mathbf{W}$, a value in the vector $\tau_{c d}$ and a value in the vector $\rho$ such that $\mathbf{W} \in \mathbb{R}^{k \times(n-j)}$ and $\tau_{c d} \in \mathbb{R}^{(n-j)}$ where $k$ and $j$ are respectively the number of DOF to control and the number of defective sensors. Instead of adjusting the size of the matrices and vectors, the algorithms presented in this paper adjust the values of $\tau_{\min }$ and $\tau_{\max }$ in such a way that the respective actuators do not receive enough power from the amplifier. This behaviour implements redundancy on parallel cable-driven haptic interfaces.

Adjusting the size of matrices and vectors has consequences on the Cartesian pose evaluation $\mathbf{P}_{p f}$ of the platform with the use of cable length $\rho$. Indeed, the measurement of cable length is obviously no longer effective. But, as with the previous Cartesian pose, it is possible to compute the theoretical length of the cable and replace the measurement by this theoretical value. Such an evaluation does not provide an accurate cartesian pose but it sufficiently protects the user and avoids resizing all the matrices and vectors in real-time. The results will show that the computed pose drifts when a cable is severed while the true pose stays constant.

\section{Safety Management Algorithm}

The following sections detail the safety strategies shown in the Fig. 1. These strategies are ordered from the low-level control (the centre of the circle) to high level haptic interaction control. This figure presents the four main strategies for human safety implemented in the control system shown in Fig. 3 and in the algorithm 1. Section 3.1 presents the algorithm designed for detecting a broken cable or a defective sensor. Section 3.2 presents the cable tension controllers used inside the control system for allowing the platform to safely go beyond its designated workspace. Finally, section 3.3 develops the control strategy for recovering from crossed cables (or interference), or when a cable intersects with a vertex of the mechanical part of the haptic interface.

\subsection{Sensor reliability}

Sensor reliability (for a fault tolerant system) is relevant to the case when a cable or a sensor can be damaged while the user is operating the haptic interface or when a sensor returns null values as shown in Fig. 5, for example. Algorithm 2 is specialized for detecting a broken cable. Two conditions are checked: the tension $\left(\tau_{m}[i] \leq 0.5 \tau_{\min }[i]\right)$ and velocity $\left(\dot{\rho}_{m}[i] \geq 0.7 \dot{\rho}_{\max }\right)$ where $\tau_{m}$ is the cable tension and 
$\rho_{m}$ is the measured cable length. A sensor could also become saturated, indicated by the fact that the acquisition board returns its maximum or minimum value for a prolonged period of time. In such conditions, the affected cable must be released from an active actuation state. Vector $\mathbf{S}_{c}[i]$ represents the state of all cables, $\varepsilon$ is the accuracy of the OTD ( $\varepsilon$ should be near zero) and vector $\mathbf{e}$ indicates a faulty sensor.

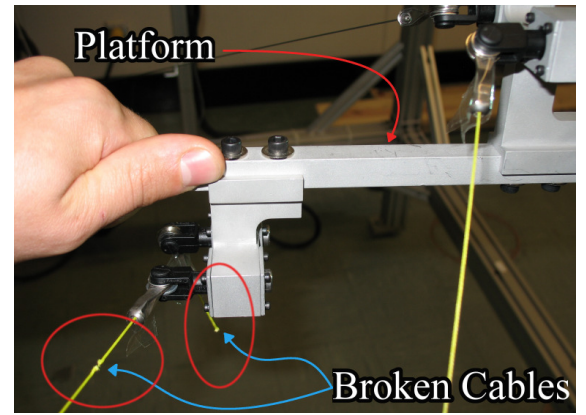

Fig. 5 Management of broken cables

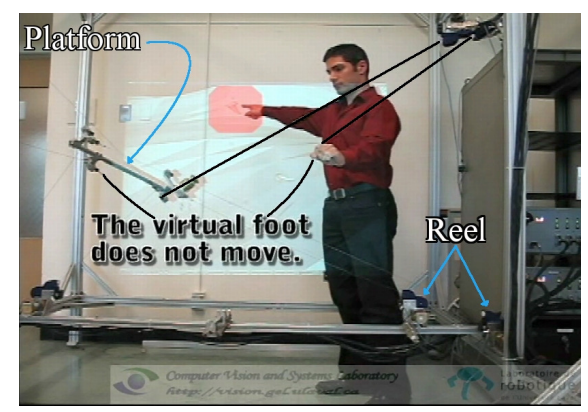

Fig. 6 Cable interference management

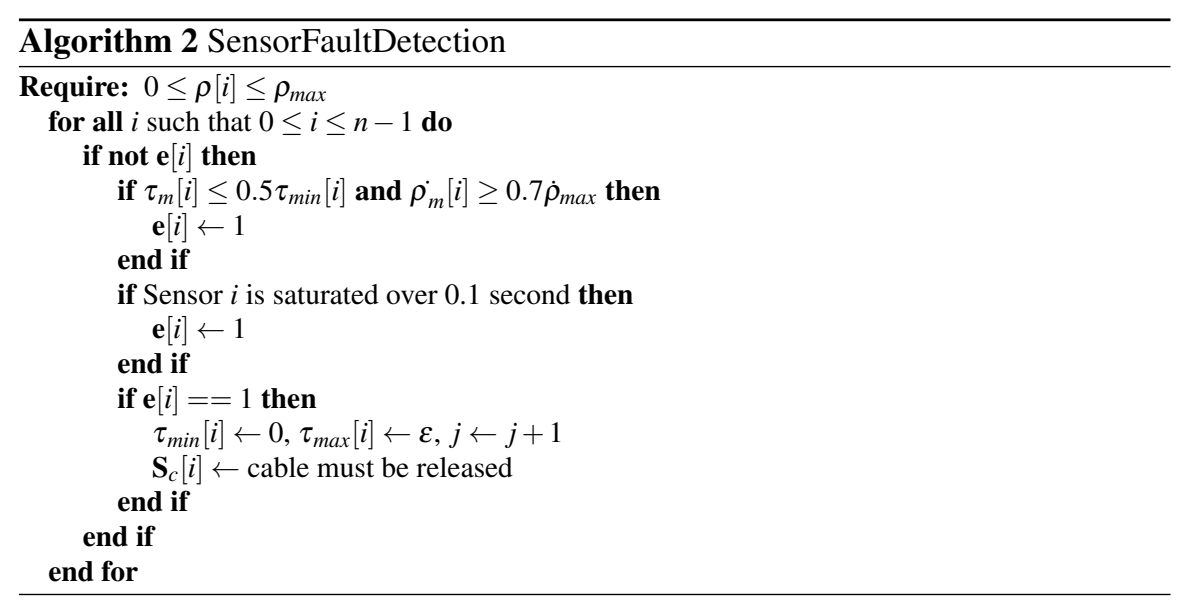

\subsection{Workspace Management}

A runtime error (RTE) switch is implemented as an open-loop controller for ensuring the security of the walker as shown in Fig 7. This RTE, controlled by vector $\mathbf{E}[i]$ for each cable tension controller $i$, allows the mechanism to go beyond its desig- 
nated workspace and return to a safe position afterwards. The OTD is deactivated and all cable tensions are maintained in an open loop system with the values $\mathbf{T}_{c d}$ at the position $(m+1) \% l$ previously computed inside the WCW. When the platform ventures outside the WCW, a resulting wrench $\mathbf{h}_{w}$ is applied on the platform that can be computed with $\mathbf{h}_{w}=\mathbf{W T}_{c d}^{(m+1) \% l}$.

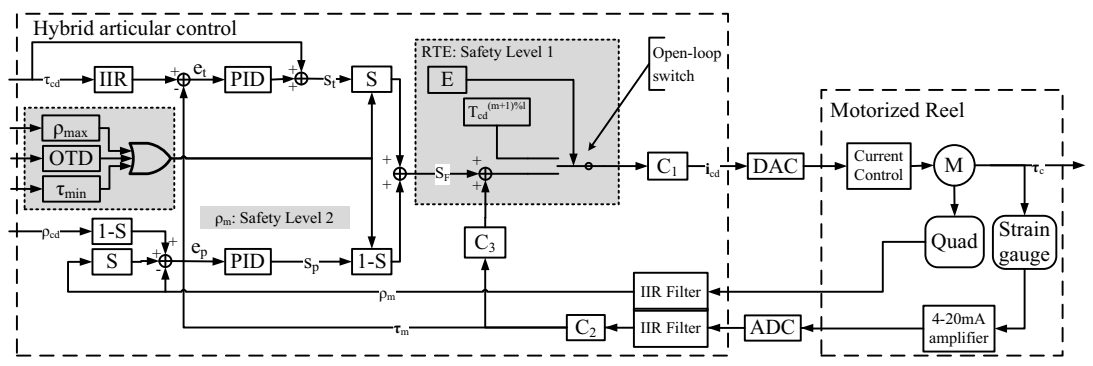

Fig. 7 Cable tension controller

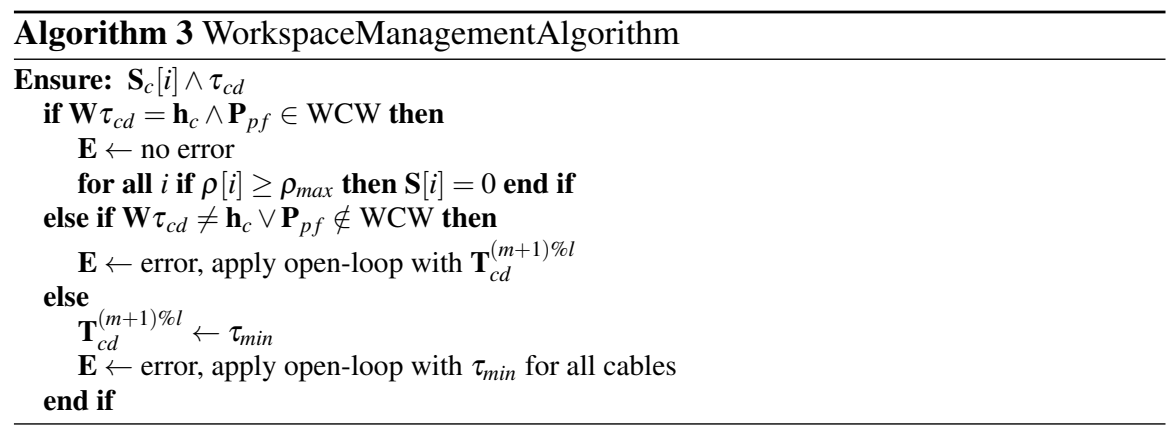

The hybrid control presented in Fig. 7 could maintain the platform at the boundary of $\rho_{\max }$ by applying cable length control. Otherwise, cable tension control is always used whenever cables are in interference, broken, or sensors are defective. The strategy used for choosing the cable length control considers three conditions: 1) maximum cable length is reached;2) $\mathbf{W} \tau_{c d} \neq \mathbf{h}_{c}$; and 3) $\tau_{m} \leq \tau_{\min }$. Note that this strategy is a second safety backup in the situation where the runtime error is not activated by the algorithms for allowing articular open-loop control.

\subsection{Mechanical Interference}

Interference between two cables can occur at any time in the course of a random trajectory such as those generated in a haptic application. This interference must be 
accounted for by choosing which cable needs to be released, or which type of control should be applied in the situation where the interfering cables cannot be released from an active actuation state. The integrity of the workspace must be verified by checking whether or not releasing a given cable keeps the platform inside the WCW. This can result in four distinct cases :

- any released cable generates a pose inside the workspace (case 1);

- cable 1 released: platform is inside the workspace (case 2);

- cable 2 released: platform is inside the workspace (case 3) and

- any released cable generates a pose outside the workspace (case 4).

Indeed, when a particular cable interferes with a mechanical component of the locomotion interface, only this cable can be released. Moreover, case 4 is still not managed. However, it is now known that the direction of the plane described by the two interfering cables can be controlled with a hybrid impedance/admittance approach by combining the developments in [8] and [14]. Currently, when case 4 is detected, the cable tensions are maintained at the $\mathbf{T}_{c d}^{(m+1) \% l}$ values in the open loop controller. Algorithm 4 thus resumes the strategy for choosing which cable must be released between two interfering cables $m$ or $n$. In this algorithm, $\mathbf{F}[i]$ flags if the platform pose is inside the WCW when cable $i$ is released.

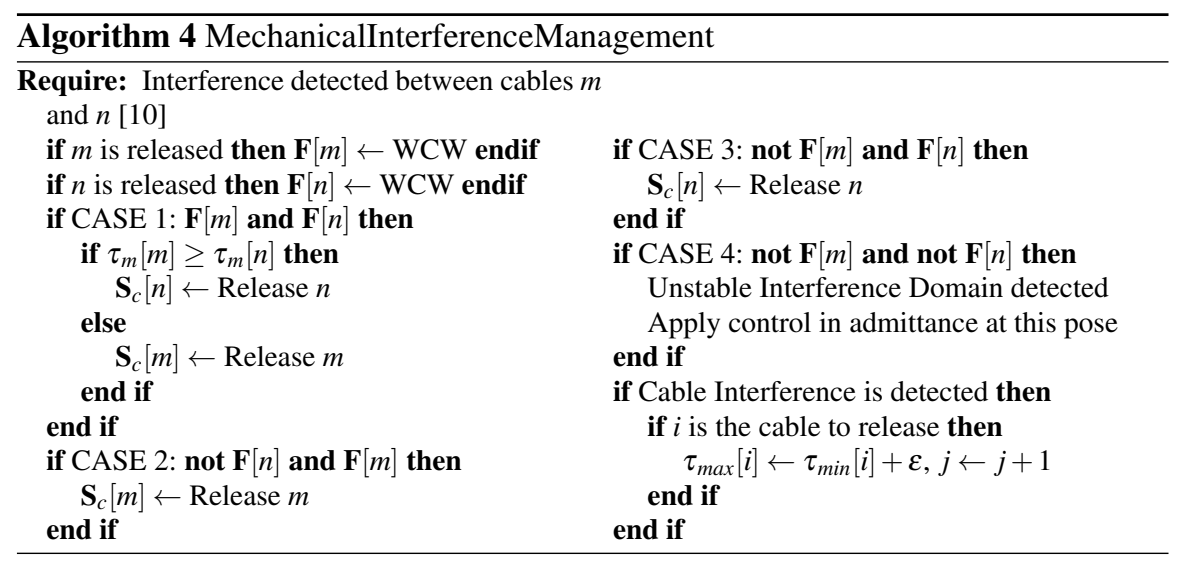

Fig. 6 shows that when a cable is released, it no longer controls the pose of the platform and thus its measured length is no longer used for computing the pose. Thus, the cable can be reeled in because it is controlled at a minimum tension since the condition $\tau_{\max }[i] \leftarrow \tau_{\min }[i]+\varepsilon$ is reached and the OTD computes tension setpoints $\tau_{c}$ with this condition. This minimum tension is computed to minimize the effect of two ambiguous criteria: reducing both cable sagging and friction at the interference point [10]. 


\section{Results and Analysis}

The aforementioned safety algorithms were applied to the control of the CableDriven Locomotion Interface prototype. This section presents some results obtained from the final control architecture for improving its reliability. The trajectories of the platforms are imposed by the user without the sensor $\left(\mathbf{h}_{a}=0\right)$ and only the weight of the platform is compensated for $\left(\mathbf{h}_{w P F} \neq 0\right.$ and $\left.\mathbf{h}_{i P F}=0\right)$. Indeed, there is only Cartesian impedance control and the platform is maintained in the free space using the OTD algorithm.

The first section described the management of sensor faults and the system reaction to a severed cable. This particular situation generates cable tension discontinuities that must be managed. The second section presents the workspace management algorithm when the user over-strains the platform outside of the workspace. Finally, additional experiments are carried out with the cable interference management algorithm in order to gain further insight on this issue presented first in [10].

\subsection{Sensor reliability}

Cable tension sensors could feasibly be damaged during system use. The defective sensor could then return the maximum or minimum value allowed by the acquisition system. It can be ignored and compensated by the other sensors. The solution to when a cable is severed can be seen in Fig 8. This figure shows that before the detection of the event, both conditions in velocity and tension are met. Some oscillations occur around zero after the cable is cut. This vibration is the characteristic Heaviside response of the strain gauge sensor used for measuring the tension inside the reel. The peak tension that is measured also results from the cantilever strain gauge response. It is possible to use this characteristic to detect the tension conditions by evaluating the exponential decreasing sinusoid coefficients with a curve fitting technique. But, this condition is only valid for the actual reel design presented in [9].

The detection delay of the event is approximately 0.1 second and the variation of the estimated pose of the platform $\mathbf{P}_{p f}$ is kept under 5 centimeters as shown in Fig 10. This variation comes from the use of the cable length for the evaluation of the platform pose. The key point is that the control system continues without failure and that the real pose of the platform is constant before and after the event. For reducing the adverse effect of a tension discontinuity shown in Fig. 9, the cable tension controllers use a setpoint IIR filter that smoothes the setpoint tensions, at the cost of reducing the bandwidth of the cable tension controllers. 

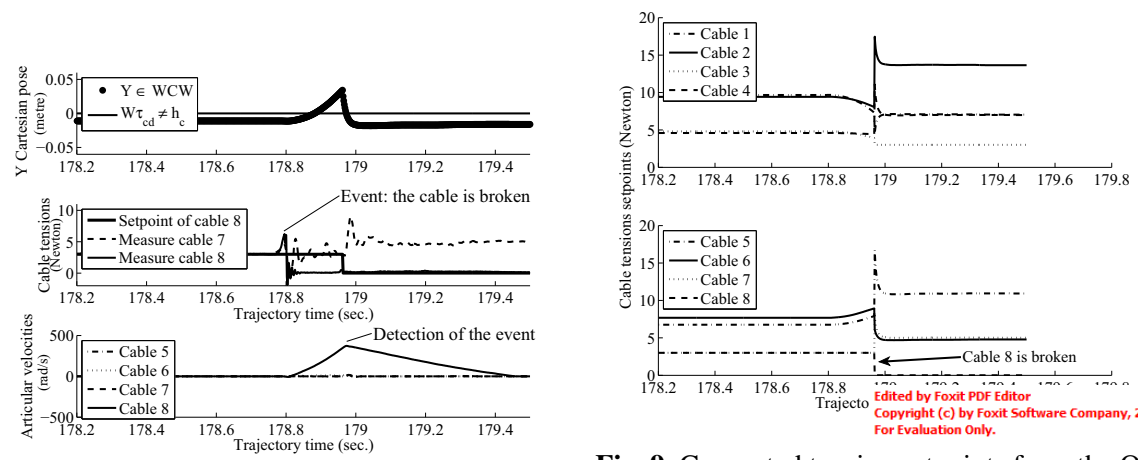

Fig. 9 Computed tension setpoints from the OpFig. 8 Cable tensions with the Y movement of timal Tension Distribution algorithm when cable the platform when cable 8 is broken

8 is broken

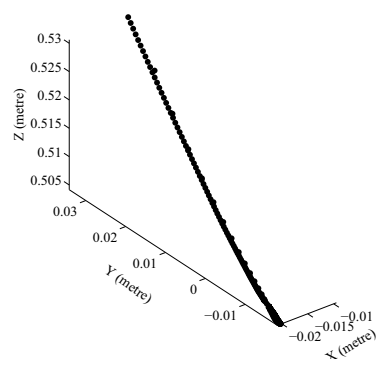

Fig. 10 Variation of the platform pose when cable 8 is broken



Fig. 11 Cartesian pose with interference between cables 2 and 6

\subsection{Workspace Management}

Fig. 12 shows the movement of the platform for the evaluation of the workspace management algorithm. When the platform goes outside the WCW, the cable tensions are kept at the constant values $\mathbf{T}_{c d}^{(m+1) \% l}$ generating a wrench $\mathbf{h}_{w}$, as seen in Fig 13, in the opposite direction of movement, ensuring that the platform will automatically return inside its workspace. Note that the cable tension discontinuities on $\tau_{c d}$ at 85.5 seconds in Fig. 12 are the result of the difference between the poses at the moment when the platform goes outside and when the platform comes back inside the WCW. Using a circular buffer $\mathbf{T}_{c d}$ to preserve past cable tensions is useful to minimize the risk that the platform should remain on the boundary of the workspace. Otherwise, when the event occurs, the cable tension setpoints are kept at their value 0.01 second before the event that is defined by the sampling frequency and the length $l$ of the buffer $\mathbf{T}_{c d}$. This timing is chosen experimentally to smoothe the transition of the solution given by the OTD for the user. 

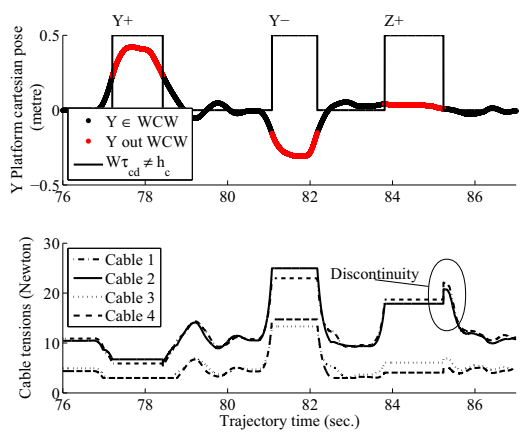

Fig. 12 Cable tensions with the $+\mathrm{Y},-\mathrm{Y}$ and $+\mathrm{Z}$

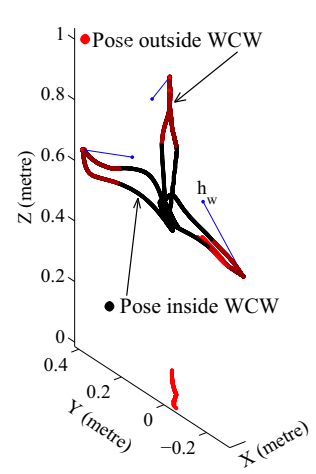

movements when the platform goes out of the Fig. 13 Cartesian pose when the platform goes workspace

outside the WCW

\subsection{Mechanical Interference Between Cables}

Fig. 11 shows the trajectory used by the cable interference management algorithm. This trajectory allows one cable interference between cables number 2 and 6. During cable interference, the platform can sometimes venture outside the WCW as shown in Fig. 14 and the cable tension setpoints are kept constant. In part 1 of the figure, between 143 and $145 \mathrm{sec}$, there is no interference and the cable tensions balance the weight of the platform. In part 2, between 145 and $149 \mathrm{sec}$, an interference is detected and cable 6 is released from its active actuation state. The setpoint of cable 6 does not drop to its minimal value $\tau_{\min }[5]$ since the OTD cannot find a valid solution for the actual pose $\mathbf{P}_{p f}$. Indeed, the workspace management algorithm maintains the cable tensions at $\mathbf{T}_{c d}^{(m+1) \% l}$. When the pose $\mathbf{P}_{p f}$ returns inside the workspace, the OTD computes new setpoints and the tension in cable 6 drops to its minimum value, as seen in the third part of the figure. Fig. 15 gives the cable tension setpoints over this trajectory.

\section{Conclusion}

When a human interacts with an active, semi-autonomous robotic mechanism, safety is a prime concern in any application to avoid injury. Sensor reliability, workspace management and mechanical interference are all critical factors within the system. The results and demonstrations presented here concern the proof that the mechanism will stay stable and safe whenever a fault occurs during operation. Thus, this paper aims to develop a strategy that allows a safer operation at all times, since the use of an emergency shutdown is not always appropriate with a cable-driven mechanism. Of course, such a mechanism does possess major problems of imple- 

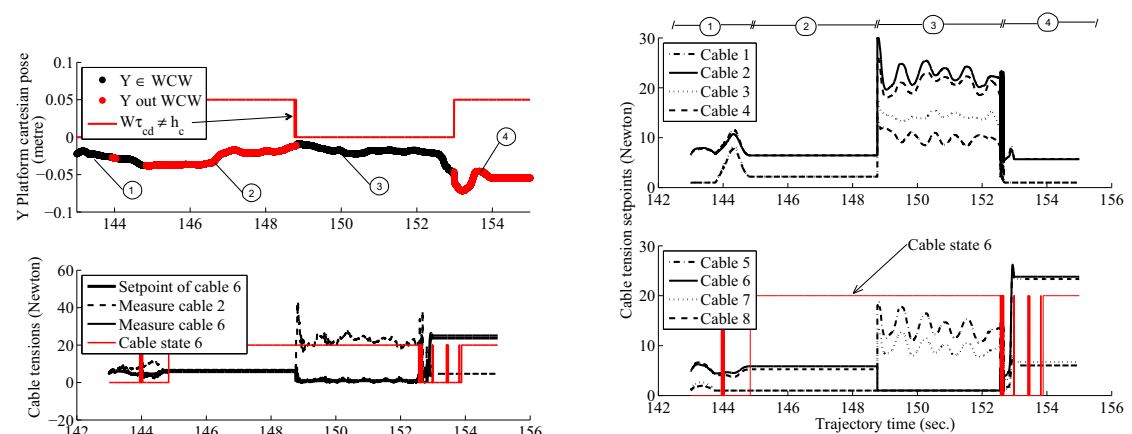

Fig. 15 Cable tension setpoints from OTD with

Fig. 14 Cable interference management results interference

mentation as a haptic device. The safety of this device is a major issue since mechanical transmission via a cable behaves differently compared to a rigid link.

The proposed software architecture includes two levels of safety implemented in Cartesian and articular hybrid controllers with different strategies for testing the overall system. However, the mechanical design plays the most important role, as the software should not be used to increase the reliability of the hardware design. In fact, these algorithms could decrease the performance of the mechanism and thus reduce the transparency of the platform's moving parts. When they are used in haptic applications, special care should be taken in investigating the trade-off between performance and safety. This paper gives further insight into the definition of a safe control algorithm applied to haptic interfaces. However, it could be extended to other systems where human-robot interaction exists.

Finally, in section 3.3, further development could be conducted for managing case 4: when both interfering cables cannot be released from their active actuation state because the platform pose is outside the WCW. Currently, in this situation, the cable tension is maintained at $\mathbf{T}_{c d}$ value.

\section{References}

1. Barrette, G., Gosselin, C.: Determination of the dynamic workspace of cable-driven planar parallel mechanisms. Journal of Mechanical Design 127(2), 242 - 8 (2005)

2. Gouttefarde, M., Gosselin, C.M.: Wrench-closure workspace of six-dof parallel mechanisms driven by 7 cables. Transactions of the Canadian Society for Mechanical Engineering 29(4), $541-552(2005)$

3. Haddadin, S., Albu-Schaffer, A., De Luca, A., Hirzinger, G.: Collision detection and reaction: A contribution to safe physical human-robot interaction. In: International Conference on Intelligent Robots and Systems, pp. 3356 - 3363. IEEE/RSJ, Nice, France (2008)

4. Hannaford, B., Ryu, J.H.: Time-domain passivity control of haptic interfaces. Transactions on Robotics and Automation 18(1), 1 - 10 (2002)

5. Iwata, H., Yano, H., Fukushima, H., Noma, H.: Circulafloor. Computer Graphics and Applications 25(1), $64-67$ (2005) 
6. Lafourcade, P.: Étude des manipulateurs parallèles à câbles, conception d'une suspension active pour soufflerie. École Nationale Supérieur de l'Aéronautique et de l'Espace (2004)

7. Lahouar, S., Ottaviano, E., Zeghoul, S., Romdhane, L., Ceccarelli, M.: Collision free pathplanning for cable-driven parallel robots. Robotics and Autonomous Systems 57(11), 1083 1093 (2009)

8. Otis, M.J.D., Mokhtari, M., Du Tremblay, C., Laurendeau, D., De Rainville, F.M., Gosselin, C.M.: Hybrid control with multi-contact interactions for 6 dof haptic foot platform on a cabledriven locomotion interface. In: Symposium on Haptics Interfaces for Virtual Environment and Teleoperator Systems, pp. 161 - 168. IEEE, Piscataway, NJ, United States (2008)

9. Otis, M.J.D., Nguyen-Dang, T.L., Laliberté, T., Ouellet, D., Laurendeau, D., Gosselin, C.: Cable tension control and analysis of reel transparency for 6-dof haptic foot platform on a cable-driven locomotion interface. International Journal of Electrical, Computer, and Systems Engineering 3(1), 16-29 (2009)

10. Otis, M.J.D., Perreault, S., Nguyen-Dang, T.L., Lambert, P., Gouttefarde, M., Laurendeau, D., Gosselin, C.: Determination and management of cable interferences between two 6-dof foot platforms in a cable-driven locomotion interface. Transactions on Systems, Man and Cybernetics, Part A: Systems and Humans 39(3), 528-544 (2009)

11. Pace, C., Seward, D.W.: A model for autonomous safety management in a mobile robot. In: International Conference on Computational Intelligence for Modelling, Control and Automation, vol. 1, pp. 1128 - 1133. IEEE, Vienna, Austria (2005)

12. Park, J., Yoon, J., Lim, Y.A., Ryu, J.: A smooth planar walking algorithm for virtual walking machine (k-walker). In: SICE-ICASE International Conference, pp. 4786-4791. Piscataway, NJ, USA (2006)

13. Perreault, S., Gosselin, C.: Cable-driven parallel mechanisms: application to a locomotion interface. Journal of Mechanical Design, Transactions 130(10), 102,301-1-8 (2008)

14. Perreault, S., Otis, M.J.D., Gosselin, C., Cardou, P.: Analysis of the interference-free constantorientation workspace of parallel cable-driven manipulators. Journal of Mechanical Design, Transactions 130(10), 102,301-1-8 (2010)

15. Roberts, R.G., Graham, T., Lippitt, T.: On the inverse kinematics, statics, and fault tolerance of cable-suspended robots. Journal of Robotic Systems 15(10), 581 - 597 (1998)

16. Ryu, J.H., Kim, Y.S., Hannaford, B.: Sampled- and continuous-time passivity and stability of virtual environments. Transactions on Robotics 20(4), 772 - 776 (2004)

17. Schmidt, H., Hesse, S., Bernhardt, R.: Safety concept for robotic gait trainers. In: International Conference on Engineering in Medicine and Biology, vol. 26 I, pp. 2703 - 2706. San Francisco, CA, United States (2004)

18. Tan, J.T.C., Duan, F., Zhang, Y., Kato, R., Arai, T.: Safety design and development of humanrobot collaboration in cellular manufacturing. In: Automation Science and Engineering, pp. 537 - 542. IEEE, Bangalore, India (2009)

19. Wischnitzer, Y., Shvalb, N., Shoham, M.: Wire-driven parallel robot: Permitting collisions between wires. International Journal of Robotics Research 27(9), 1007 - 1026 (2008)

20. Yoon, J., Park, J., Ryu, J.: A symmetric walking cancellation algorithm of a foot-platform locomotion interface. In: International Conference on Humanoid Robots, pp. 668 - 673. IEEERAS, Piscataway, NJ, USA (2008)

21. Zheng, Y., Liu, X.: Optimal tension distribution of wire-driven parallel manipulators. Jixie Gongcheng Xuebao/Chinese Journal of Mechanical Engineering 41(9), 140 - 145 (2005) 